\title{
Modelo de pronóstico de reoperación en cirugía abdominal
}

\author{
Caridad de Dios Soler-Morejón ${ }^{1 *}$, Tomás Ariel Lombardo-Vaillant ${ }^{2}$, Teddy Osmín Tamargo-Barbeito ${ }^{3}$, Manu Malbrain ${ }^{4}$
}

Resumen: Introducción: En presencia de complicaciones infecciosas intrabdominales postoperatorias, la decisión de reoperar es todavía difícil para el cirujano actuante. Los modelos matemáticos representan una buena ayuda al diagnóstico en estas condiciones. Método: Estudio prospectivo observacional de 300 pacientes post-cirugía abdominal ingresados en la unidad de cuidados intensivos del Hospital Calixto García desde enero de 2008 a enero de 2010. Los pacientes fueron aleatoriamente separados (2:1) en dos grupos; estimación (GE) y validación (GV). En el GE se desarrollaron tres modelos estadísticos para la reoperación, que fueron validados en el GV .Estos modelos incluyeron variables, que en estudios anteriores demostraron su utilidad en el pronóstico, como el índice predictivo de reoperación aguda (ARPI) y la presión intrabdominal (PIA) Resultados: El modelo ARPI-PIA fue el mejor de los tres modelos, según el estadígrafo Hosmer-Lemeshow (calibración C=9,976 p=0.267, discriminación área bajo la curva ROC=0,989 IC 95\% 0,976-1,000). Conclusión: La inclusión de la PIA junto al ARPI en un modelo matemático puede aumentar la certeza del pronóstico de reoperación en presencia de complicaciones infecciosas intrabdominales tras cirugía abdominal. Este modelo puede ser de utilidad en situaciones de recursos diagnósticos limitados.

Palabras clave: infección intrabdominal; presión intrabdominal; índice predictivo de reoperación aguda; modelo pronóstico; reoperación abdominal.

Abstract: Background: The decision of re-operating after abdominal surgery is still difficult, especially whenan intra-abdominal infectious complication is present. Mathematical models represent good diagnosis aid. Methods: A prospective observational study was conducted with 300 patients after abdominal surgery consecutively admitted at the intensive care unit of the "CalixtoGarcía Hospital" from January 2008 to January 2010. The patients were randomly separated (2:1) into estimation and validation groups. Three models for re-operation were developed in the estimation group by logistic regression, using some factors that demonstrated their usefulness in previous studies, for example, the acute re-operation predictive index (ARPI) and the intra-abdominal pressure (IAP). Afterwords, the models were validated on the other group. Results: Acute re-operation predictive index-intraabdominal pressure (ARPI-IAP) model was the best of the three models, with an excellent calibration by the Hossmer-Lemeshow goodness-of fit statistic ( $C=9,976 p=0,267)$ discrimination ( $\mathrm{AUC}=0,98995 \% \mathrm{Cl}$ 0,976-1,000). Conclusion: The combination of IAP with ARPI in a mathematical model can add accuracy to the prediction of re-operation related to intra-abdominal infectious complications in patients after abdominal surgery. This model is recommended in conditions of limited diagnostic resources.

Keywords: intra-abdominal infection; intra-abdominal pressure; acute re-operation predictive index; prognostic model; abdominal re-operation

Fecha de envío: 1 de Febrero de 2016 - Fecha de aceptación: 16 de Diciembre de 2016

\section{Introducción}

No existe un consenso general sobre cómo y cuándo reoperar a un paciente con complicaciones infecciosas intrabdominales después de cirugía abdominal. Pese a la disponibilidad de avanzadas técnicas de imágenes, todavía resulta difícil la decisión de reoperar y frecuentemente, aun los cirujanos expertos se encuentran ante un verdadero reto (Pusajo et al., 1993; Lombardo Vaillant et al., 2009). En general, los criterios clínicos y la experiencia personal, con la ayuda de las pruebas diagnósticas, son los recursos más comúnmente empleados para decidir la estrategia quirúrgica,

(1) Unidad de Cuidados Intensivos, Hospital Hermanos Ameijeiras La Habana, Cuba.

(2) Departamento de Cirugía, Hospital Calixto García, La Habana, Cuba.

(3) Departamento de Bioestadística, Hospital Hermanos Ameijeiras, La Habana, Cuba.

(4) Intensive Care Unit, ZNA Stuivenberg, Antwerp, Belgium.

Autor de correspondencia: csoler@infomed.sld.cu 
el ingreso en la unidad de cuidados intensivos o evaluar el pronóstico. A pesar de las evidencias de su utilidad, los sistemas para el pronóstico y los índices de gravedad no son ampliamente usados (De Waele et al., 2007).

Hasta el presente, no existe un índice ideal, pero los que están vigentes al menos permiten obtener información adicional sobre el diagnóstico y el pronóstico y consecuentemente una de las principales ventajas de su aplicación es aumentar la calidad de los cuidados de salud que se brindan. Más aún, los sistemas de pronóstico pueden ser integrados con algunos factores de riesgo para el desarrollo de modelos de predicción. Sin duda, la modelación es una de las herramientas más usadas en la actualidad para analizar y evaluar el objeto de estudio en una realidad condicionada (Cook, 2008).

El índice predictivo de reoperación abdominal (Acute Reoperation Predictive Index, ARPI, por sus siglas en inglés) (Pusajo et al., 1993), es un sistema de ayuda que incluye un árbol de decisión de utilidad para el tratamiento quirúrgico de las complicaciones. Aunque en nuestra experiencia su especificidad es del100\%, su sensibilidad no es tan elevada (Lombardo Vaillant et al., 2009). Por otra parte, la presión intrabdominal elevada (PIA) es un conocido factor independiente relacionado con las complicaciones y la mortalidad en la cirugía abdominal (Khan et al., 2010). Por tanto, puede plantearse la hipótesis de que la combinación de la PIA con el ARPI en un modelo matemático puede añadir certeza al pronóstico de reoperación relacionado con la presencia de complicaciones infecciosas intrabdominales en pacientes después de cirugía abdominal.

\section{Método}

Se desarrolló un estudio prospectivo observacional con una cohorte de 300 pacientes, que después de cirugía abdominal, emergente o electiva, fueron ingresados consecutivamente en la unidad de cuidados intensivos (UCI) del Hospital Calixto García, desde enero de 2008 a enero de 2010 por 48 horas o más. Se excluyeron los casos de embarazo, reoperación por causas no infecciosas, reoperación programada, pacientes sin posibilidades de medir la PIA y los fallecidos en las primeras 48 horas de operado.

Los pacientes fueron separados aleatoriamente en dos grupos (2:1): estimación (GE) y validación (GV). La variable dependiente fue reoperación y las variables independientes fueron la edad, ARPI, PIA, tipo de cirugía (electiva o de emergencia), y la duración de la cirugía. El ARPI fue calculado diariamente a partir del primer día del posoperatorio, durante la evaluación matutina. Para minimizar la variabilidad interobservador, tres o más especialistas hicieron el cálculo del ARPI de manera independiente y cegada, y posteriormente se obtuvo un valor promedio para guiar la conducta clínica de acuerdo con el árbol de decisiones. El valor tomado en consideración fue el obtenido el día en que se decidió la intervención en los casos necesarios de acuerdo con el árbol de decisiones ya mencionado. En los pacientes no reoperados, se reconsideró retrospectivamente al egreso de la $\mathrm{UCl}$, el mayor valor calculado durante el postoperatorio según la pauta establecida en el trabajo original (Pusajo et al., 1993).

Se midió la PIA en cada paciente según la técnica de Cheatham y Safcsak (Cheatham \& Safcsak, 1998) y las recomendaciones de la Sociedad Mundial del Síndrome de Compartimiento Abdominal (World Society of Abdominal Compartment Syndrome, WSACS) (Kirkpatrick et al., 2013); pero en lugar de emplear un transductor de presiones se colocó en el sistema de drenaje de orina una columna de agua con una escala en centímetros ( $\mathrm{cmH2O})$.

Se realizaron dos mediciones al final de la espiración, con un intervalo de 6 horas, durante las primeras 24 horas de ingreso del paciente. Las mediciones se hicieron por enfermeras entrenadas en la realización de la técnica bajo la supervisión del especialista. Se empleó un volumen intravesical de $25 \mathrm{ml}$ de solución salina. El cero fue colocado a nivel de la línea media axilar con el paciente en posición supina, con la cresta ilíaca superior como punto de referencia. Cada valor de PIA se obtuvo mediante manometría (cmH2O) y se recalculó en $\mathrm{mm}$ de $\mathrm{Hg},(1 \mathrm{cmH} 2 \mathrm{O}=0.74 \mathrm{mmHg})$. Los cuatro valores obtenidos en cada paciente fueron promediados $y$ los resultados se introdujeron en una base de datos.

También fue calculado el APACHE II (Knaus et al., 1981) durante las primeras 24 horas de ingreso en la unidad de cuidados intensivos.

La reoperación se decidió de acuerdo con la evaluación clínica, el árbol de decisión y el valor de la PIA. Se consideró la existencia de hipertensión intrabdominal (HIA), si la PIA estuvo por encima de $12 \mathrm{mmHg}$ en dos mediciones consecutivas (Kirkpatrick et al., 2013).

Se consideró como "estándar de oro" para la confirmación de complicación infecciosa intrabdominal los hallazgos durante la reoperación. Cada paciente fue seguido de forma individual hasta su egreso del hospital (vivo o fallecido).El protocolo fue aprobado por el comité de ética local y se solicitó el consentimiento informado a los pacientes o a su persona mas allegada antes de iniciar la inclusión en el estudio. La medición de la PIA no interfirió con otros procedimientos diagnósticos o terapéuticos, de acuerdo con los principios de la Declaración de Helsinki (World Medical Association Declaration of Helsinki, 2008). 


\section{Control y análisis estadístico de los datos}

Las medidas de tendencia central y de dispersión usadas fueron la media, desviación estándar e intervalos de confianza 95\% (Cl) para las variables cuantitativas, mientras que se emplearon frecuencias y porcentajes para las variables cualitativas. Se empleó el test de Chi cuadrado con corrección de Yates de continuidad siempre que fuera apropiado identificar diferencias entre variables categóricas. La prueba " $t$ " de Student se empleó para comparar las diferencias entre las medias de variables continuas entre grupos en condiciones basales.

Se desarrollaron tres modelos de regresión logística para analizar la influencia de un grupo de variables sobre la probabilidad de reoperar:

Modelo I (ARPI): variables independientes: edad, género, ARPI, tipo de cirugía y duración de la cirugía.

Modelo II (PIA): variables independientes: edad, género, PIA, tipo de cirugía y duración de la cirugía.

Model III (ARPI-PIA): variables independientes: edad, género, PIA, ARPI, tipo de cirugía y duración de la cirugía.

Las historias clínicas seleccionadas fueron distribuidas de forma aleatoria en dos grupos: 200 para estimación (GE) y 100 para validación (GV). En el GE se estimaron los parámetros del modelo de regresión logística. El GV permitió chequear la capacidad real de predicción de los modelos para nuevos pacientes (Figura 1).

Se calcularon los Odds ratios (OR) y los IC 95\% para evaluar la fortaleza de alguna posible asociación que surgiera. La curva de características para el operador (COR) para evaluar la habilidad de discriminación de cada modelo para los pacientes con y sin reoperación, de acuerdo con los valores de sensibilidad y especificidad para cada punto de la curva. El poder discriminativo del modelo se consideró excelente si el área bajo la curva $\mathrm{ROC}(\mathrm{ABC})$ fue de $>0.80$, muy buena si >0.75, y buena si >0.70 (Swets, 1988). La calibración de cada modelo fue valorada mediante la prueba de bondad de ajuste de Hosmer-Lemeshow. Según este estadígrafo, una $\mathrm{p}>0,05$ indica una calibración aceptable del modelo.

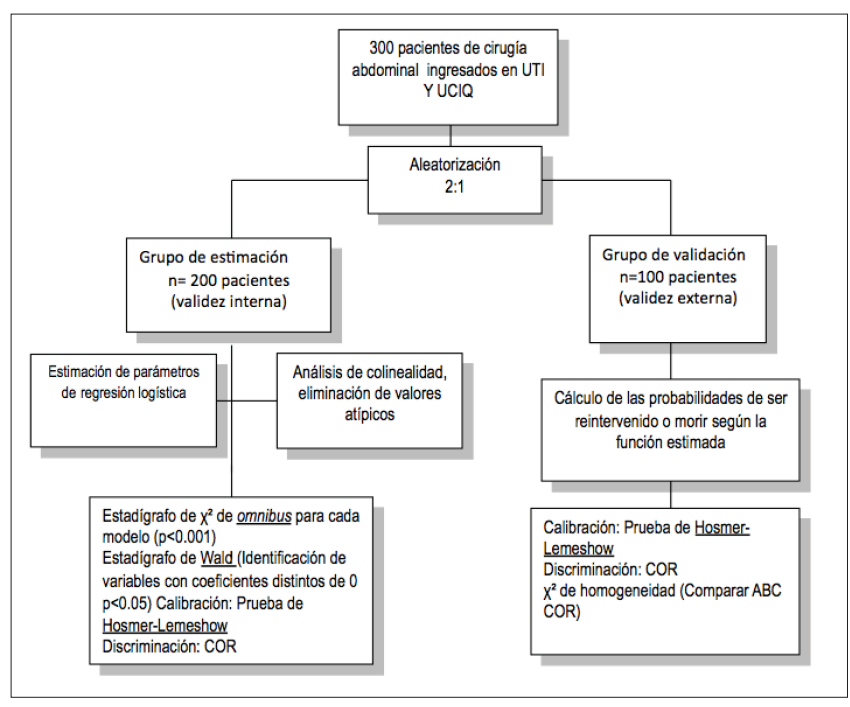

Figura 1. Análisis estadístico. COR: curva de características para el operador

Para organizar, validar y analizar los datos recolectados se empleó el Statistical Package for Social Sciences (SPSS para Windows versión $11.5^{\circledR}$ software SPSS Inc., Chicago, IL, USA). Se consideró un nivel de significación de $5 \%$ en todas las pruebas de hipótesis.

\section{Resultados}

Del total de pacientes incluidos (300), el 88\% recibió cirugía de emergencia y el resto, cirugía electiva (Figura 2). Fueron reoperados 102 pacientes (34\%) y hubo 24 pacientes fallecidos (23.52\%). La mortalidad fue de cero en el grupo de los pacientes que no fueron reoperados (198 pacientes).

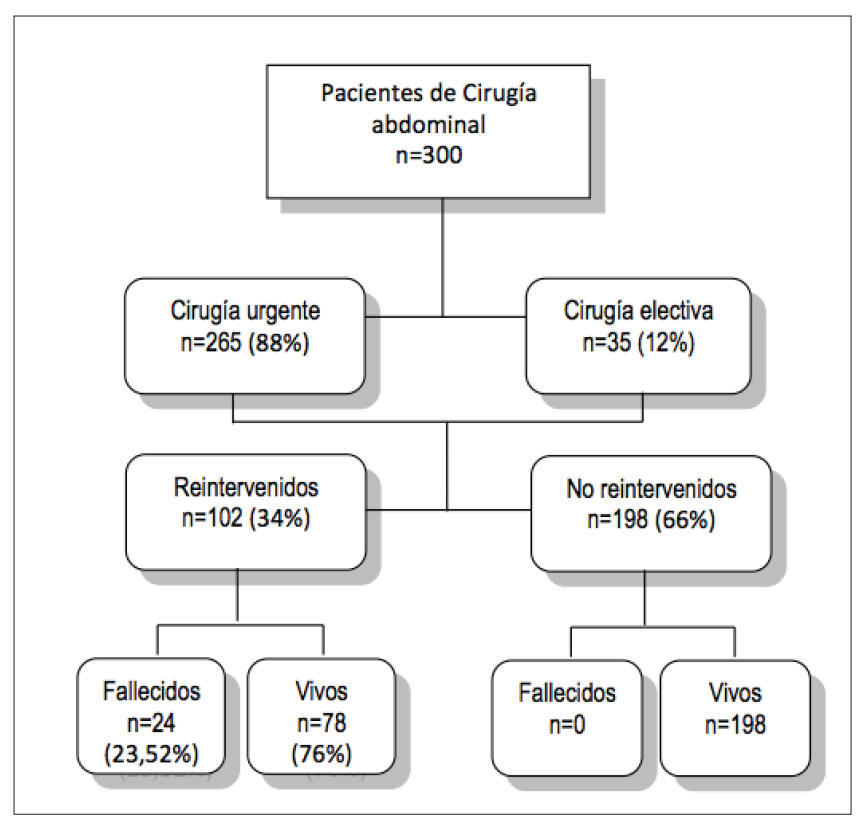

Figura 2. Caracterización de la población de estudio. 
Los GE y GV fueron similares respecto a género, comorbilidad, reoperación, malignidad, valores de PIA, APACHE II y mortalidad, pero el GV tenía una edad media superior (Tabla 1). La mortalidad total fue del $23,52 \%$.

Tabla 1. Características de los pacientes quirúrgicos en los grupos de estimación y validación

\begin{tabular}{|c|c|c|c|c|}
\hline & & $\begin{array}{c}G E \\
n=200\end{array}$ & $\begin{array}{c}\mathrm{GV} \\
\mathrm{n}=100\end{array}$ & $\mathrm{p}$ \\
\hline \multicolumn{2}{|c|}{ Masculinos } & $126(63,0 \%)$ & $62(62,0 \%)$ & \multirow{2}{*}{$0,966^{\circ}$} \\
\hline \multicolumn{2}{|c|}{ Femeninos } & $74(37,0 \%)$ & $38(38,0 \%)$ & \\
\hline \multicolumn{2}{|l|}{ Edad } & $53,4 \pm 19,8$ & $58,2 \pm 19,8$ & $0,048^{b}$ \\
\hline \multirow{2}{*}{ Comorbilidad } & Sí & $192(96,0 \%)$ & $96(96,0 \%)$ & \multirow{2}{*}{0,755} \\
\hline & No & $8(4,0 \%)$ & $4(4,0 \%)$ & \\
\hline \multirow{2}{*}{ Reoperación } & Sí & 71 (35,5\%) & $31(31,0 \%)$ & \multirow{2}{*}{0,518} \\
\hline & No & $129(64,5 \%)$ & $69(69,0 \%)$ & \\
\hline \multirow{2}{*}{ Malignidad } & Yes & $9(4,5 \%)$ & $4(4,0 \%)$ & \multirow{2}{*}{$0,920^{\mathrm{a}}$} \\
\hline & No & 191 (95,5\%) & $96(96,0 \%)$ & \\
\hline \multicolumn{2}{|l|}{ PIA } & $12,2 \pm 5,4$ & $12,1 \pm 5,4$ & $0,821^{b}$ \\
\hline \multicolumn{2}{|c|}{ APACHE II } & $16,5 \pm 6,0$ & $16,0 \pm 5,9$ & $0,506^{b}$ \\
\hline \multicolumn{2}{|c|}{ Mortalidad } & $17(8.5 \%)$ & $7(7.0 \%)$ & $0,821^{a}$ \\
\hline
\end{tabular}

a: Chi cuadrado con corrección de Yates b: "t"Student

El hallazgo más común en la primera operación fue la apendicitis, seguido de la obstrucción intestinal y la colecistitis aguda.

Los procedimientos quirúrgicos utilizados fueron fundamentamente la resección parcial, anastomosis y derivaciones según el diagnóstico intraoperatorio. En un segundo tiempo, cuando fue posible, se realizó anastomosis término-terminal para solucionar las derivaciones realizadas.

Los hallazgos más frecuentes en los pacientes reoperados fueron los abscesos intrabdominales y las dehicencias de suturas con peritonitis (23,5\%) En 12 pacientes reoperados no se encontraron hallazgos patológicos. En la tabla 2 se muestran los tres modelos estadísticos desarrollados.
Tabla 2. Modelos estadísticos desarrollados

\begin{tabular}{|c|c|}
\hline Modelo & Ecuación \\
\hline I: ARPI & $\begin{array}{c}\mathrm{P}(\text { reoperación })=1 /\left(1+\mathrm{EXP}\left(3.557+0.001^{*} \text { edad }+\right.\right. \\
0.914^{*} \text { género } \\
+0.017^{*} \text { duración de la cirugía- } 0.304^{*} \mathrm{ARPI}-1.356^{*} \text { tipo } \\
\text { de cirugía })\end{array}$ \\
\hline II:PIA & $\begin{array}{l}\mathrm{P}(\text { reoperación })=1 /\left(1+\operatorname{EXP}\left(14.448-0.031^{*} \text { edad- }\right.\right. \\
0.111^{*} \text { género } \\
+0.007^{*} \text { duración de la cirugía- } 0.222^{*} \text { tipo de } \\
\left.\text { cirugía- } 0.958^{*} \mathrm{PIA}\right)\end{array}$ \\
\hline III: ARPI-PIA & $\begin{array}{l}\mathrm{P}(\text { reoperación })=1 /\left(1+\mathrm{EXP}\left(15.835-0.041^{*} \text { edad- }\right.\right. \\
0.263^{*} \text { género- } 0.895^{*} \mathrm{PIA}+0.029^{*} \text { duración de la } \\
\left.\text { cirugía- } 0.311^{*} \mathbf{A R P I}-0.042^{*} \text { tipo de cirugía }\right)\end{array}$ \\
\hline
\end{tabular}

Los tres modelos tuvieron una excelente discriminación, como se aprecia en la tabla 3. Sin embargo, mostraron diferencias en cuanto a los coeficientes. En el modelo I (ARPI), las variables ARPI, género y duración de la cirugía mostraron coeficientes significativamente diferentes de cero $(p<0,05)$. El OR para el ARPI fue de 1,355 (IC 95\%, $245-1,475) p=0,000$. ARPI fue la variable más influyente sobre la probabilidad de ser reoperado (coeficiente estándar 2,656). Este modelo tuvo una excelente discriminación, con un $A B C>0.92$ en los dos grupos.

En el modelo II (PIA), solo la PIA mostró coeficientes significativamente diferentes de cero $(p<0,05)$. OR=2,606 (IC 95\%1,859-3,652) $p=0$, 00 . En esta cohorte de pacientes, en relación con los coeficientes estandarizados, la variable de más influencia sobre la probabilidad de reoperación fue la PIA. Este modelo tuvo también una excelente $A B C(>0.95)$ en los dos grupos.

En cuanto al modelo III (ARPI-PIA) PIA, ARPI y duración de la cirugía fueron las variables cuyos coeficientes fueron diferentes de cero. Los OR relacionados con el ARPI, la PIA y la duración de la cirugía fueron de 1,365, 2,448 y 0,971 respectivamente. Según los coeficientes estandarizados, en esta cohorte de pacientes la variable de mayor influencia sobre la probabilidad de reoperar fue la PIA, seguida por el ARPI. Este modelo tuvo la mejor discriminación con un $A B C>0.97$ en los dos grupos.

Respecto a la validación interna, en los tres modelos estimados (GE) se observó un buen ajuste (Hosmer-Lemeshow $\mathrm{p}>0,05$ ) y una excelente calibración. En el GV, los tres modelos no mostraron una probabilidad significativa asociada a la prueba de Hosmer- Lemeshow ( $p>0,05$ ), lo que implica una excelente calibración. (Tabla3). La discriminación fue también catalogada como excelente. 
Tabla 3. Calibración y discriminación de los tres modelos para la reoperación según los índices pronósticos en pacientes post cirugía abdominal

\begin{tabular}{|c|c|c|c|c|c|c|c|c|c|}
\hline \multirow[t]{3}{*}{ Modelo } & & \multicolumn{4}{|c|}{$\begin{array}{c}\text { Validación Interna } \\
\text { (GE) }\end{array}$} & \multicolumn{4}{|c|}{$\begin{array}{l}\text { Validación Externa } \\
\text { (GV) }\end{array}$} \\
\hline & \multicolumn{2}{|c|}{ Hosmer-Lemeshow } & \multicolumn{2}{|c|}{ COR } & \multicolumn{2}{|c|}{ Hosmer-Lemeshow } & \multicolumn{2}{|c|}{ COR } & \\
\hline & C & $\mathbf{p}$ & ABC & IC (95\%) & C & $\mathbf{p}$ & ABC & IC (95\%) & \\
\hline ARPI & & 8,167 & 0,417 & 0,934 & $0,902-0,967$ & 1,441 & 0,230 & 0,925 & $0,875-0,976$ \\
\hline PIA & & 4,024 & 0,855 & 0,978 & $0,963-0,993$ & 0,039 & 0,843 & 0,951 & $0,914-0,989$ \\
\hline ARPI-PIA & & 9,976 & 0,267 & 0,989 & $0,976-1,000$ & 0,580 & 0,446 & 0,973 & $0,948-0,998$ \\
\hline
\end{tabular}

En la figura 3 puede apreciarse la comparación entre los tres modelos. El modelo ARPI-PIA ofrece la mejor curva COR y la mayor $A B C(0,973, C l 0,948-0,998)$ por lo que resulta el de máxima utilidad para la predicción de la probabilidad de reoperar. Aunque los tres modelos poseen una excelente discriminación y calibración, la diferencia entre las tres $A B C$ fue significativa (Chi cuadrado $10,172, p=0,001)$.

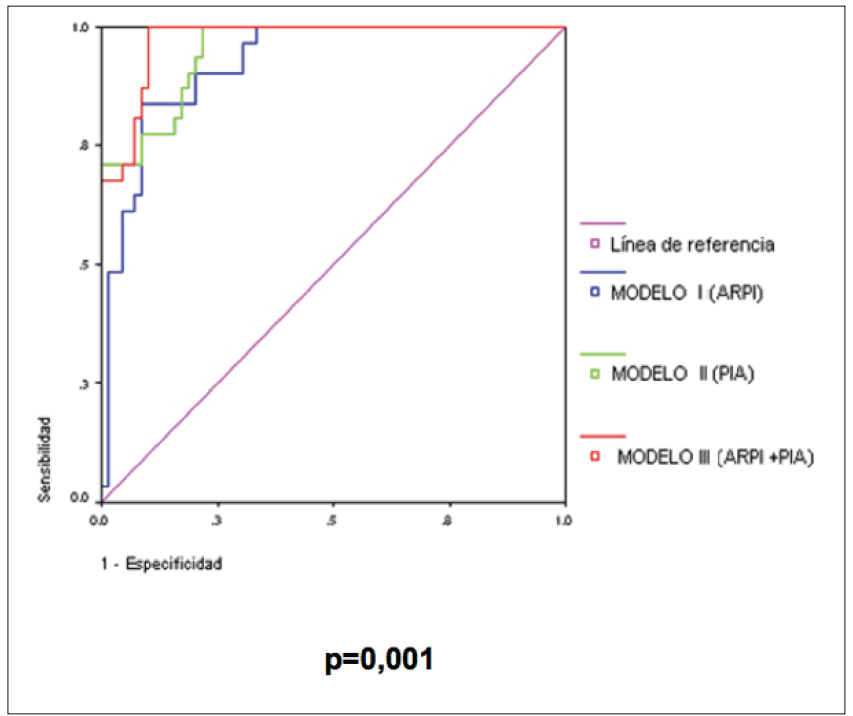

Figura 3. Comparación de las curvas COR

\section{Discusión}

Para el desarrollo de los modelos se incluyeron entre los factores seleccionados dos índices de puntuación que en estudios previos fueron relacionados con la necesidad de reoperar (Pusajo et al., 1993; Koperna \& Schulz, 2000; Filgueiras Ramos et al., 2001; Shinkawa et al., 2003; Pisanu et al., 2004; Komatsu et al., 2005; Makela et al., 2005; Rakic et al., 2005; Yeh et al., 2005; Reyes de la Paz et al., 2007; Lombardo Vaillant et al., 2009; Panhofer et al., 2009; Pera, 2011).
Pese a lo señalado por autores como Pusajó y colaboradores en sus series de pacientes (Pusajo et al., 1993), en esta cohorte la edad no fue reconocida como un factor de riesgo independiente asociado a la reoperación. Tampoco el género constituyó en este estudio un factor de riesgo significativo para la reoperación. Solo en el modelo I (ARPI), el género masculino aparece como un factor de riesgo. Los hallazgos previos que han sido reportados en relación con el género son controvertidos y no demuestran una clara asociación con la necesidad de reoperar (Wacha et al., 1987; Schroder et al., 1998; Aydin et al., 2006; Biondo et al., 2006).

La comorbilidad no quedó incluida como un factor independiente para la reoperación en los modelos desarrollados, a pesar de que no fue escasa su presencia en esta cohorte. Probablemente las características de la población estudiada pudieran explicar este resultado.

Algunos autores señalan que la malignidad resulta una condición adversa para el pronóstico de los pacientes quirúrgicos. En estos casos resulta más frecuente la dehicencia de sutura con peritonitis, la progresión a peritonitis bacteriana asociada o no a la radioterapia previa al procedimiento quirúrgico y la existencia de eventos adversos durante el transoperatorio (Lee et al., 2008). Sin embargo, en este estudio la malignidad no fue reconocida como un factor de riesgo para la reoperación, lo cual pudiera relacionarse con la baja prevalencia de esta enfermedad en la muestra estudiada.

Tampoco el tipo de cirugía constituyó un factor significativo para la reoperación en esta cohorte de pacientes, aunque en general se considera su asociación con la morbilidad y mortalidad posquirúrgica, sobre todo, en el caso de la cirugía de emergencia (Okubo et al., 2008), debido a disfunción y falla de órganos y sistemas. No obstante, Van Ruler y colaboradores argumentan que la razón principal no depende del tipo de cirugía, sino de la causa y su magnitud, y que el tipo de cirugía se relaciona con la fisiopatología (van Ruler et al., 2007). 
Se conoce que el desarrollo de modelos puede revelar ciertas relaciones que no se muestran claramente en otras condiciones (Montesinos-López \& Hernández Suárez, 2007). En este estudio, la duración de la cirugía posee un efecto protector en relación con la necesidad de reoperar (OR=0,971 IC $95 \% 0,971-0,996 \mathrm{p}=$ $0,025)$ con coeficientes ajustados y estandarizados negativos (Coef $(b)=-0,029$ Coef (b) $\left.E^{a}=-1,296\right)$. Este resultado resulta aparentemente paradójico en una cohorte de pacientes donde más del $80 \%$ de las cirugías fueron de emergencia (265 pacientes), y donde se excluyeron aquellos casos cuyas reoperaciones fueron programadas, independientemente de las características de su primera intervención o de su APACHE II score. La duración del acto quirúrgico depende de la combinación de un conjunto de factores subjetivos y objetivos, relacionados con indicadores de estructura y proceso (Donabedian, 2005) que incluye la curva de aprendizaje de los cirujanos noveles (Haji et al., 2009; Kim et al., 2010). Sin embargo, Gil-Bona J y colaboradores plantean que son precisamente las complicaciones intraoperatorias que el paciente puede desarrollar (y no la duración de la cirugía) las que constituyen un factor determinante asociado a la mortalidad quirúrgica (Gil-Bonaa et al., 2010).

Aunque los tres modelos poseen un excelente poder de discriminación y calibración, existen diferencias significativas entre ellos y es posible establecer un nivel jerárquico. El modelo III ARPI-PIA ofrece la mejor discriminación, seguido por el modelo PIA. Este modelo identificó mejor a aquellos pacientes con una probabilidad real de presentar complicaciones infecciosas intrabdominales.

La asociación de ambos sistemas de ayuda, según el principio booleano (la regla de la "Y") permite interpretar ambos parámetros en paralelo, aumenta la sensibilidad y especificidad del diagnóstico, y sirve de apoyo a la evaluación clínica (Weinstein et al., 2005). Sin duda, la inclusión de la PIA en el modelo matemático le confirió mayor exactitud al pronóstico de reoperación en esta cohorte de pacientes poscirugía abdominal en presencia de infecciones intrabdominales.

Hasta el presente, no se conocen reportes de previas aplicaciones de esta combinación de variables integradas en modelos estadísticos. Se destaca el informe de Pusajó y col., que propusieron el ARPI (Pusajo et al., 1993) y de Basu \& Pai (2008), que aplicó el incremento de la PIA $(p=0,055)$ como criterio de reoperación en pacientes con peritonitis bacteriana secundaria, pero no existen referentes de la asociación de esas variables en un modelo matemático.

Este estudio posee algunas limitaciones. Se trata de un estudio realizado en un solo centro, donde el número de reoperados y la mortalidad fueron elevados en comparación con reportes de otros autores, pero se trata de pacientes graves, con APACHE II por encima de 13 puntos, con una mortalidad dentro de límites permisibles si se tiene en cuenta la gravedad de la peritonitis que presentaron. La evaluación clínica, elemento esencial durante la toma de decisiones, fue bien tenida en cuenta durante la conformación de los modelos, sin embargo, no se realizó ninguna comparación con las imágenes diagnósticas disponibles, ya que se tomaron como estándar de oro los hallazgos en el transoperatorio.

\section{Conclusiones}

La combinación de diferentes factores pronósticos relacionados en los modelos estadísticos presentados contribuye a aumentar la certeza de la predicción de la necesidad de reoperar en pacientes críticos poscirugía abdominal. El modelo ARPI - PIA fue el más exacto, seguido por el modelo PIA. Sin duda, la integración de la PIA al modelo ARPI tiene un efecto aditivo para la predicción de la reoperación abdominal en esta cohorte de pacientes críticos. El modelo ARPI-PIA puede ser de especial utilidad en centros con limitados recursos tecnológicos.

\section{Contribuciones y reconocimientos:}

CS participó en el diseño, análisis de la base de datos, discusión de los resultados y confección del manuscrito. TAL participó en el diseño, análisis y discusión de los resultados y en la revisión del manuscrito. TOT participó en el diseño, realizó el análisis estadístico y participó en el análisis de los resultados y revisión del manuscrito. MM participó en la discusión de los resultados y revisión final del manuscrito.

Los autores declaran que no existen conflictos de interés. No se recibió ninguna fuente de financiamiento para la realización de este estudio.

\section{Referencias}

Aydin C, Altaca G, Berber I, Tekin K, Kara M \& Titiz I. (2006). Prognostic parameters for the prediction of acute gangrenous cholecystitis. Journal of hepato-biliary-pancreatic surgery 13, 155-159.

Basu A \& Pai DR. (2008). Early elevation of intra-abdominal pressure after laparotomy for secondary peritonitis: a predictor of relaparotomy? World journal of surgery 32, 1851-1856.

Biondo S, Ramos E, Fraccalvieri D, Kreisler E, Rague JM \& Jaurrieta E. (2006). Comparative study of left colonic Peritonitis Severity Score and Mannheim Peritonitis Index. The British journal of surgery 93, 616-622. 
Cheatham MLMD \& Safcsak KRN. (1998). Intraabdominal Pressure: A Revised Method for Measurement. Journal of the American College of Surgeons 186, 368-369.

Cook NR. (2008). Statistical evaluation of prognostic versus diagnostic models: beyond the ROC curve. Clinical chemistry 54, 17-23.

De Waele JJ, De laet I \& Malbrain ML. (2007). Rational intraabdominal pressure monitoring: how to do it? Acta clinica Belgica 62 Suppl 1, 16-25.

Donabedian A. (2005). Evaluating the quality of medical care. 1966. The Milbank quarterly 83, 691-729.

Filgueiras Ramos B, Bembibre Taboada R, Corona Martínez LA \& Soler Morejón C. (2001). Monitoreo de la presión intraabdominal (PIA) en el paciente quirúrgico grave. Revista Cubana de Cirugía 40, 18-23.

Gil-Bonaa J, Sabatéa A, Miguelena Bovadillab JM, Adroera R, Kooa M \& Jaurrieta E. (2010). Valor de los índices de Charlson y la escala de riesgo quirúrgico en el análisis de la mortalidad operatoria. Cirugía Española 88, 174-179.

Haji A, Khan A, Haq A \& Ribeiro B. (2009). Elective laparoscopic cholecystectomy for surgical trainees: predictive factors of operative time. The surgeon : journal of the Royal Colleges of Surgeons of Edinburgh and Ireland 7, 207-210.

Khan S, Verma AK, Ahmad SM \& Ahmad R. (2010). Analyzing intra-abdominal pressures and outcomes in patients undergoing emergency laparotomy. Journal of emergencies, trauma, and shock 3, 318-325.

Kim SY, Hong SG, Roh HR, Park SB, Kim YH \& Chae GB. (2010). Learning curve for a laparoscopic appendectomy by a surgical trainee. Journal of the Korean Society of Coloproctology 26, 324-328.

Kirkpatrick AW, Roberts DJ, De Waele J, Jaeschke R, Malbrain ML, De Keulenaer B, Duchesne J, Bjorck M, Leppaniemi A, Ejike JC, Sugrue M, Cheatham M, Ivatury R, Ball CG, Reintam Blaser A, Regli A, Balogh ZJ, D'Amours S, Debergh D, Kaplan M, Kimball E, Olvera C \& Pediatric Guidelines Sub-Committee for the World Society of the Abdominal Compartment S. (2013). Intra-abdominal hypertension and the abdominal compartment syndrome: updated consensus definitions and clinical practice guidelines from the World Society of the Abdominal Compartment Syndrome. Intensive care medicine 39, 1190-1206.

KnausWA, Zimmerman JE, Wagner DP, Draper EA \& Lawrence DE. (1981). APACHE-acute physiology and chronic health evaluation: a physiologically based classification system. Critical care medicine 9, 591-597.
Komatsu S, Shimomatsuya T, Nakajima M, Amaya H, Kobuchi T, Shiraishi S, Konishi S, Ono S \& Maruhashi K. (2005). Prognostic factors and scoring system for survival in colonic perforation. Hepato-gastroenterology 52, 761-764.

Koperna T \& Schulz F. (2000). Relaparotomy in peritonitis: prognosis and treatment of patients with persisting intraabdominal infection. World journal of surgery 24, 32-37.

Lee WS, Yun SH, Roh YN, Yun HR, Lee WY, Cho YB \& Chun HK. (2008). Risk factors and clinical outcome for anastomotic leakage after total mesorectal excision for rectal cancer. World journal of surgery 32, 1124-1129.

Lombardo Vaillant TA, Soler Morejón C, Lombardo Vaillan J \& Casamayor Laime Z. (2009). Aplicación del índice predictivo de reintervención abdominal en el diagnóstico de complicaciones infecciosas intraabdominales. Revista Cubana de Medicina Militar 38, 0-0.

Makela JT, Kiviniemi H \& Laitinen S. (2005). Prognostic factors of perforated sigmoid diverticulitis in the elderly. Digestive surgery 22, 100-106.

Montesinos-López O \& Hernández Suárez CM. (2007). Modelos matemáticos para enfermedades infecciosas. Salud Pública en México 49, 218-226.

Okubo R, Yajima K, Sakai Y, Kido T, Hirano K, Musha N, Tsubono T \& Hatakeyama K. (2008). Short-and long-term outcomes of surgery for diffuse peritonitis in patients 80 years of age and older. Surgery today $\mathbf{3 8}, \mathbf{4 1 3 - 4 1 9 .}$

Panhofer P, Izay B, Riedl M, Ferenc V, Ploder M, Jakesz R \& Götzinger P. (2009). Age, microbiology and prognostic scores help to differentiate between secondary and tertiary peritonitis. Langenbeck's Archives of Surgery 394, 265-271.

Pera M. (2011). Dehiscencia de anastomosis y recurrencia del cáncer colorrectal: una asociación que refuerza el valor pronóstico del cirujano en el resultado oncológico. Cirugía Española 89, 2225.

Pisanu A, Cois A \& Uccheddu A. (2004). Surgical treatment of perforated diverticular disease: evaluation of factors predicting prognosis in the elderly. International surgery $\mathbf{8 9}, \mathbf{3 5 - 3 8 .}$

Pusajo JF, Bumaschny E, Doglio GR, Cherjovsky MR, Lipinszki Al, Hernandez MS \& Egurrola MA. (1993). Postoperative intra-abdominal sepsis requiring reoperation. Value of a predictive index. Archives of surgery 128, 218-222; discussion 223. 
Rakic M, Popovic D, Rakic M, Druzijanic N, Lojpur M, Hall BA, WiIliams BA \& Sprung J. (2005). Comparison of on-demand vs planned relaparotomy for treatment of severe intra-abdominal infections. Croatian medical journal 46, 957-963.

Reyes de la Paz A, Lombardo Vaillant A \& Rubio Olivares DY. (2007). Valor predictivo de la presión intra-abdominal en el diagnóstico de complicaciones posoperatorias abdominales. Revista Cubana de Medicina Militar 36.

Schroder J, Kahlke V, Staubach KH, Zabel P \& Stuber F. (1998). Gender differences in human sepsis. Archives of surgery 133, 1200-1205.

Shinkawa H, Yasuhara H, Naka S, Yanagie H, Nojiri T, Furuya Y, Ariki $\mathrm{K} \&$ Niwa H. (2003). Factors affecting the early mortality of patients with nontraumatic colorectal perforation. Surgery today 33, 13-17.

Swets JA. (1988). Measuring the accuracy of diagnostic systems. Science 240, 1285-1293.

van Ruler O, Mahler CW, Boer KR, Reuland EA, Gooszen HG, Opmeer BC, de Graaf PW, Lamme B, Gerhards MF, Steller EP, van Till JW, de Borgie CJ, Gouma DJ, Reitsma JB, Boermeester MA \& Dutch
Peritonitis Study G. (2007). Comparison of on-demand vs planned relaparotomy strategy in patients with severe peritonitis: a randomized trial. Jama 298, 865-872.

Wacha H, Linder M, Feldmann U, Wesch G, Gundlach E \& Steifensand R. (1987). Mannheim peritonitis index-prediction of risk of death from peritonitis: construction of a statistical and validation of an empirically based index. Theor Surg 1, 169-177.

Weinstein S, Obuchowski NA \& Lieber ML. (2005). Clinical evaluation of diagnostic tests. AJR American journal of roentgenology 184, 14-19.

World Medical Association Declaration of Helsinki. (2008). Ethical Principles for Medical Research Involving Human Subject. In 59th WMA General Assembly. Seúl, Corea.

Yeh CY, Changchien CR, Wang JY, Chen JS, Chen HH, Chiang JM \& Tang R. (2005). Pelvic drainage and other risk factors for leakage after elective anterior resection in rectal cancer patients: a prospective study of 978 patients. Annals of surgery 241, 9-13. 